\section{Are podoplanin and ezrin involved in the invasion process of the ameloblastomas?}

\author{
Y.F. Costa, ${ }^{1}$ K.C. Tjioe, ${ }^{1}$ S. Nonogaki, ${ }^{2}$ \\ F.A. Soares, ${ }^{3}$ J.R. Pereira Lauris, ${ }^{1}$ \\ D. Tostes Oliveira \\ 'Department of Stomatology, Area of \\ Pathology, Bauru School of Dentistry, \\ University of São Paulo, Bauru \\ ${ }^{2}$ Adolfo Lutz Institute, Pathology \\ Division, São Paulo \\ ${ }^{3}$ Department of Pathology, A.C. Camargo \\ Cancer Hospital, São Paulo, Brazil
}

\section{Abstract}

The association between podoplanin and ezrin in the process of odontogenic tumors invasion has been suggested, but was not studied yet. Our purpose was to investigate the relationship between podoplanin and ezrin expressions in the odontogenic epithelium of ameloblastomas. Forty-seven ameloblastomas were analyzed by immunohistochemistry using anti-podoplanin and anti-ezrin antibodies. The expressions of both proteins were evaluated using a score method and the comparison and association between these proteins were verified, respectively, by Wilcoxon Signed-Rank test and by Spearman's rank correlation coefficient, using a statistical significance level of 0.05 . The majority of tumors (87.2\%) exhibited strong membranous expression of podoplanin in the peripheral cells. Cytoplasmic expression of ezrin in the peripheral cells of ameloblastomas was stronger than its membranous expression. No statistically significant correlation was observed between podoplanin and ezrin. However, there was statistically significant difference between membranous podoplanin and membranous ezrin expressions, between cytoplasmic podoplanin and membranous ezrin expressions, and between cytoplasmic podoplanin and cytoplasmic ezrin expressions. There was no statistical difference between membranous podoplanin and cytoplasmic ezrin expressions. These results suggest a synergistic role of both proteins in the process of invasion of ameloblastomas.

\section{Introduction}

Emerging studies of podoplanin (PDPN) have shown that this transmembrane glycoprotein plays a role in cellular movements with a high demand of actin cytoskeleton remodeling, ${ }^{1-3}$ a primordial condition to the development of benign and malignant tumors. The overexpression of PDPN by oral cancer cells, especially in the invasive front of the tumor has been associated to poor clinical outcome and lymph nodal metastasis. ${ }^{4-10}$

Particularly in benign odontogenic tumors, including ameloblastomas, keratocystic odontogenic tumors, calcifying cystic odontogenic tumors, adenomatoid odontogenic tumors and odontomas, ${ }^{11-22}$ the PDPN expression by neoplastic cells has been basically found in the periphery of odontogenic islands and cords, regions responsible by the local invasion of the tumor. Furthermore, it was previously showed $^{11,18,22}$ that membranous and cytoplasmic immunostaining of podoplanin in follicular ameloblastomas are predominantly seen in the peripheral columnar cells of tumoral islands while the reticulum stellate-like cells, located in the central areas, do not present positivity for this protein. An interesting and recent study showed that PDPN expression is increased in the tumoral invasive front of recurrent ameloblastomas when compared with the central region. ${ }^{22}$ Additionally, the expression of PDPN basically is restricted to the basal and supra-basal layers of the cystic lining in unicystic ameloblastomas. ${ }^{18,22}$ These data suggest the participation of PDPN in the local invasion of ameloblastomas but the mechanisms by which this protein contributes for this process are still a mystery. Recent investigations showed that podoplanin increases cell motility through interaction of this protein with ERM (ezrin, radixin and moesin) proteins family and subsequent modulation of the Rho proteins and actin cytoskeleton.,23,24 According to Martin-Villar et $a l .,{ }^{2}$ the PDPN directly binds ezrin, activating RhoA, a protein belonging to the family of GTPases. This linkage induces the phosphorylation of ezrin, promoting the actin cytoskeleton rearrangement in vitro. ${ }^{2}$ Therefore, the ezrin and podoplanin proteins are involved in maintenance of the cellular shape and polarity through cytoskeleton. ${ }^{25-27}$ Clinical investigations showed that overexpression of ezrin by malignant epithelial cells in oral squamous cell carcinoma has been associated to poor clinical outcome and lower survival rate of the patients, ${ }^{28-31}$ similar to findings of the podoplanin in cancer studies mentioned above. So far, neither the expression of ezrin in the ameloblastomas, nor its relation with podoplanin expression have been studied. Thus, the aim of this study was to contribute with the knowledge of the interaction of ezrinpodoplanin in ameloblastomas, particularly in the process of local invasion of these tumors.
Correspondence: Dr. Denise Tostes Oliveira, Bauru School of Dentistry, University of São Paulo, Department of Stomatology, Area of Pathology, Alameda Octávio Pinheiro Brisolla 975, 17012-901 Bauru, SP, Brazil.

Tel. +55.14.32358251 - Fax: +55.14.32234679.

E-mail: denisetostes@usp.br

Key words: Odontogenic tumor, ameloblastoma, podoplanin, ezrin, adhesion molecule.

Funding: this study was supported by the Fundação de Amparo à Pesquisa do Estado de São Paulo (FAPESP) with grants \#2012/08278-5 and \#2012/13411-6.

Conflict of interest: the authors declare no conflict of interest.

Received for publication: 11 September 2014. Accepted for publication: 29 December 2014.

This work is licensed under a Creative Commons Attribution NonCommercial 3.0 License (CC BYNC 3.0).

(C) Copyright Y.F. Costa et al., 2015

Licensee PAGEPress, Italy

European Journal of Histochemistry 2015; 59:2451 doi:10.4081/ejh.2015.2451

\section{Materials and Methods}

\section{Ethics statement}

This study was approved by the Ethic Committee in Human Research of the Bauru School of Dentistry, University of São Paulo (CAAE \#03924312.0.0000.5417).

\section{Patient and tumor samples}

Paraffin-embedded tissue specimens of ameloblastomas were collected from the archives of the Laboratory of Pathology in the Department of Stomatology of Bauru School of Dentistry, University of São Paulo, between 1980 and 2009 . These samples were previously analyzed by Tjioe et al. ${ }^{18}$ and updated to 2013 . The inclusion criteria were: i) patients with diagnosis of solid ameloblastoma or of unicystic ameloblastoma, based on the clinical, radiographic and microscopic data; ii) representative sections of the tumor for microscopic analysis. The diagnosis of ameloblastoma was reviewed according to the World Health Organization histological classification of odontogenic tumors. ${ }^{32}$ A total of 47 ameloblastomas were selected for immunohistochemistry analysis of the podoplanin and ezrin expressions.

\section{Podoplanin and ezrin immuno- expressions in ameloblastomas}

Formalin-fixed, $4 \mu \mathrm{m}$ sections of ameloblas- 
tomas were obtained for podoplanin and ezrin immunoexpressions by odontogenic epithelium cells. After antigen retrieval using $10 \mathrm{mM}$ citrate buffer, $\mathrm{pH} 6.0$, in a domestic pressure cooker (model Eterna 41/2 L; Nigro, Araraquara, Brazil) for $4 \mathrm{~min}$, endogenous peroxidase activity was blocked by incubating in $3 \% \mathrm{H}_{2} \mathrm{O}_{2}$ for 20 min. Each ameloblastoma section was incubated overnight at $4^{\circ} \mathrm{C}$ in a $1: 200$ dilution of the primary monoclonal anti-podoplanin antibody (D2-40 clone, Dako North America, Inc., Carpinteria, CA, USA, code\#3619-1) or in a 1:1000 dilution of the anti-ezrin antibody (3C12 clone, LabVision Co., Freemont, CA, USA, code\#MS-661P). Primary antibody dilutions were made in a phosphate-buffered saline (PBS)/bovine serum albumin (\#A2153, Sigma-Aldrich, St. Louis, M0, USA) solution. After the primary antibody incubation, each section was incubated using the Advance HRP Link System (Dako Inc., Carpinteria, CA, USA; \#K4067) for $30 \mathrm{~min}$ at $37^{\circ} \mathrm{C}$. Both antibodies (podoplanin and ezrin) were detected using 3,3'diaminobenzidine tetrahydrochloride (Sigma-Aldrich; \#D-5637). Sections were counterstained with Mayer s hematoxylin before being dehydrated and cover slipped. Palatine tonsils and gut were used as the positive control for anti-podoplanin and anti-ezrin antibodies, respectively. The primary antibody was omitted during immunohistochemical staining for the negative control. Computerassisted analysis of 10 invasive tumor front fields (magnification 400x) in each ameloblastoma sample was performed to evaluate the cytoplasmic and/or membranous immunoreactivity of podoplanin and ezrin by odontogenic epithelial cells. The tumor invasive front was defined according to Piffkò et al. ${ }^{33}$ as the 3-6 tumor cell layers or detached tumor cell groups at the advancing edge of the tumors.

The images in each ameloblastoma were digitally captured with a camera (Axiocam MR3; Zeiss, Jena, Germany) attached to a light microscope (Axioskop 2 Plus; Zeiss) and recorded by Axiovision software (Axiovision 4.6; Zeiss). A combined score ${ }^{34}$ for PDPN and ezrin expressions by odontogenic epithelial cells was based on: i) the intensity of the immunostaining by odontogenic epithelial cells ( 0 , absent; 1 , weak; 2 , moderate; 3 , strong; 4 , very strong); and ii) the percentage of positive odontogenic epithelial cells $(0,0 \%$ positive cells; $1,<25 \%$ positive cells; $2,25-50 \%$ positive cells; $3,50-75 \%$ positive cells; $4,>75 \%$ positive cells). The final immunostaining score was determined by the sum of i) + ii) and it ranged from 0 to 8 (0, absent; 1-4, weak; 5-8, strong). Membranous and cytoplasmic podoplanin and ezrin expressions by odontogenic epithelial cells of ameloblastomas were analyzed separately.

\section{Statistical analysis}

The correlation between PDPN and ezrin expressions by odontogenic epithelial cells of the ameloblastomas was calculated using Spearman's rank correlation coefficient. The comparison between the cytoplasmic and membranous expressions of PDPN and ezrin in ameloblastomas was verified by Wilcoxon Signed-Rank test. The level of significance was set at $5 \%$ for all tests.

\section{Results}

\section{Clinical features}

Table 1 summarizes the distribution of tumors according to the histopathological type and clinical features. The patients' ages ranged from 9 to 68 years, with a median of 32.2 years. It was observed a predominance of females (68.1\%) in relation to males (31.9\%). The most common location was the posterior mandible (55.4\%; 26 tumors), followed by concomitant posterior and anterior mandible in 5 tumors, anterior mandible (4 tumors), posterior maxilla (3 tumors) and anterior maxilla (1 tumor). In 8 ameloblastomas it was not possible to register the locations of the tumors.
Table 1. Clinical features and histopathological classification of the 47 ameloblastomas studied.

\begin{tabular}{lcc} 
Variable & N & $\%$ \\
Gender & & \\
$\quad$ Female & 32 & 68.1 \\
$\quad$ Male & 15 & 31.9 \\
Age & & \\
0 to 19 & 8 & 17 \\
20 to 39 & 20 & 42.5 \\
40 to 59 & 6 & 12.8 \\
$\geq 60$ & 3 & 6.4 \\
Unknown & 10 & 21.3 \\
Location & & \\
Posterior mandible & 26 & 55.4 \\
Anterior mandible & 4 & 8.5 \\
Posterior maxilla & 3 & 6.4 \\
Anterior maxilla & 1 & 2.1 \\
Anterior and posterior mandible & 5 & 10.6 \\
$\quad$ Unknown & 8 & 17 \\
Histopathological classification & & \\
Follicular & 21 & 44.7 \\
Plexiform & 14 & 29.8 \\
Unicystic (luminal) & 7 & 14.9 \\
Unicystic (mural) & 4 & 8.5 \\
Mixed & 1 & 2.1 \\
Total & 47 & 100 \\
\hline
\end{tabular}
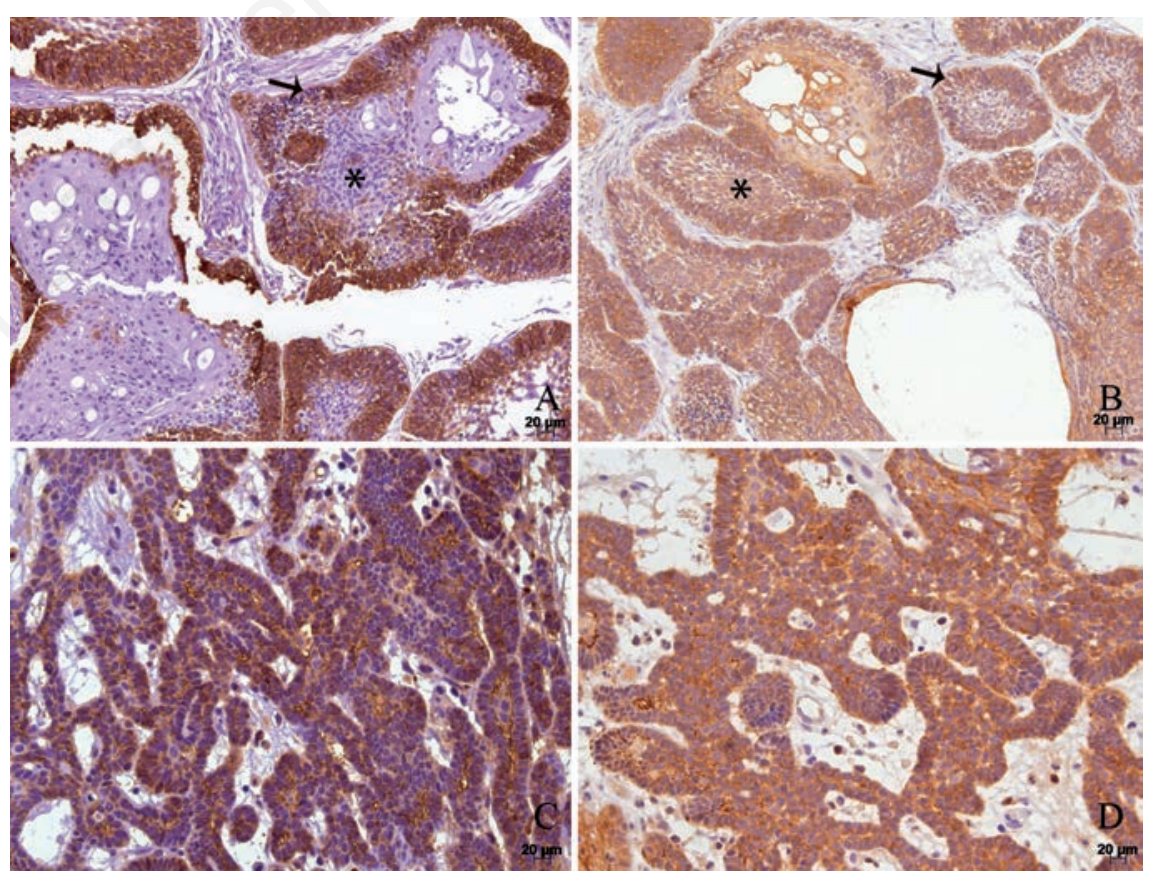

Figure 1. Immunohistochemical reactivities for podoplanin $(A, C)$ and ezrin $(B, D)$ in follicular ameloblastoma with acanthomatous areas $(A, B)$ and plexiform ameloblastoma (C,D). A) Podoplanin positivity is confined to the outer odontogenic cells (arrows) while the reticulum stellate-like (asterisks) and acanthomatous cells are negative for the protein. B) Ezrin is positive in all odontogenic cells and structures, namely outer, inner and acanthomatous cells. C) In plexiform ameloblastoma, a homogeneous expression of podoplanin (C) as well as of ezrin (D) is found in the whole tumor. 


\section{Podoplanin expression by odonto- genic epithelial cells of ameloblas- tomas}

The podoplanin expression was detected in the lymphatic vessels (internal positive control immunostaining) and in the odontogenic epithelial cells of the ameloblastomas.

The expression of podoplanin in follicular ameloblastomas was predominantly observed in the peripheral cells of tumoral islands, while the central cells exhibited weak/absent immunostaining (Figure 1A). On the other hand, the plexiform ameloblastomas showed the podoplanin expression in the peripheral cells as well as in the central part of some tumors (58\% of cases) (Figure 1C). Acanthomatous areas (Figure 1B) and focal areas of granular cells did not present immunoreaction to anti-podoplanin antibody. The expression of podoplanin was quite variable in the unicystic ameloblastomas. Few tumors presented a restricted immunostaining to the basal layer (Figure 1D), while in others its positivity was spread throughout the extension of the tumoral odontogenic epithelium.

Membranous and cytoplasmic immunostaining of podoplanin were analyzed in each ameloblastoma, and was predominantly positive in membranous area. These results can be seen in Table 2 . There was no statistically significant difference between membranous and cytoplasmic podoplanin expressions by peripheral odontogenic epithelial cells of the ameloblastoma ( $\mathrm{P}=0.157)$. Furthermore, most central cells of the tumors (84.8\%) showed weak or absent podoplanin expression.

\section{Ezrin expression by odontogenic epithelial cells of ameloblastomas}

In a general way, the expression of ezrin was positive for both peripheral and central cells of follicular, plexiform and unicystic tumors (Figure $2 \mathrm{~A}-\mathrm{D}$ ). The acanthomatous areas showed strong expression of this protein in the tumors (Figure 2D).

Contrarily from podoplanin, there was a predominance of cytoplasmic ezrin expression by odontogenic epithelial cells of the ameloblastomas in comparison with membranous expression, and this difference was statistically significant $(\mathrm{P}=0.000)$, as illustrated in Table 3. Sixtythree percent of tumors exhibited strong positivity for ezrin in the odontogenic epithelial cells located in the center of cords and islands of ameloblastomas (Figure 3B).

\section{Association between podoplanin and ezrin expressions in ameloblas- tomas}

In order to verify whether podoplanin expression was directly or inversely associated to ezrin expression, the Spearman's rank correlation test was employed. However, there was no statistically significant correlation between membranous and/or cytoplasmic expressions of both antibodies ( $\mathrm{P}>0.05$, Table 4$)$. On the other hand, the Wilcoxon Signed-Rank test demonstrated statistically significant difference between membranous podoplanin and membranous ezrin expressions $(\mathrm{P}=0.000)$; cytoplasmic podoplanin and membranous ezrin expressions $(\mathrm{P}=0.000)$ and between cytoplasmic podoplanin and cytoplasmic ezrin

Table 2. Podoplanin immunoexpression by odontogenic epithelial cells in ameloblastomas.

\begin{tabular}{lccccc} 
& \multicolumn{2}{c}{ Membranous } & \multicolumn{2}{c}{ Podoplanin } & \multicolumn{2}{c}{ Cytoplasmic } & P \\
& N & $\%$ & N & $\%$ & \\
Absent & 6 & 12.8 & 1 & 2.1 & \\
Weak & 0 & 0 & 1 & 2.1 & $0.157^{*}$ \\
\hline Strong & 41 & 87.2 & 45 & 95.8 & \\
Total & 47 & 100 & 47 & 100 & \\
\hline
\end{tabular}

*Wilcoxon test; statistical significant at $\mathrm{P}<0.05$.

Table 3. Ezrin immunoexpression by odontogenic epithelial cells in ameloblastomas.

\begin{tabular}{|c|c|c|c|c|c|}
\hline & & & & & \\
\hline & & & & & P \\
\hline & $\mathbf{N}$ & $\%$ & $\mathbf{N}$ & $\%$ & \\
\hline Absent & 29 & 61.7 & 5 & 10.6 & \\
\hline Weak & 4 & 8.5 & 5 & 10.6 & $0.000^{*}$ \\
\hline Strong & 14 & 29.8 & 37 & 78.8 & \\
\hline Total & 47 & 100 & 47 & 100 & \\
\hline
\end{tabular}

*Wilcoxon test; statistical significant at $\mathrm{P}<0.05$.

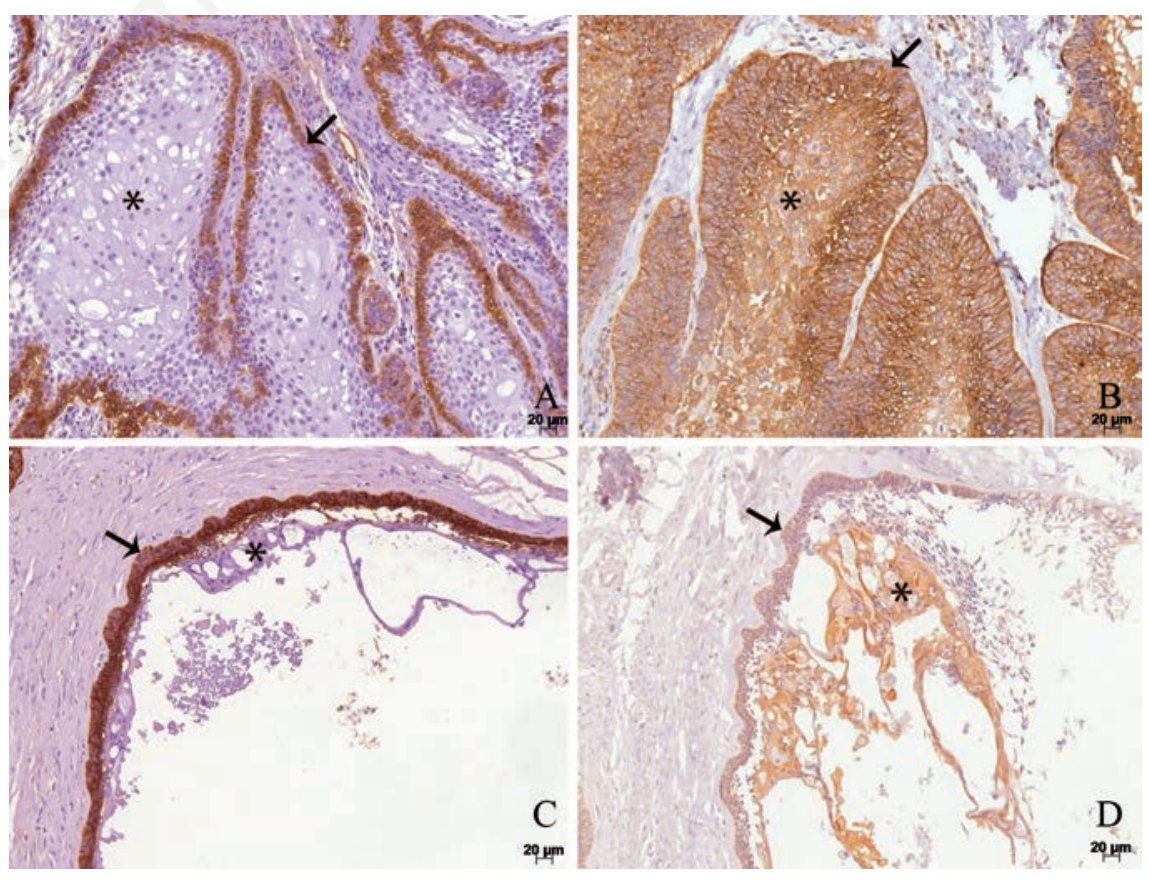

Figure 2. Immunohistochemical reactivities for podoplanin $(A, C)$ and ezrin $(B, D)$ in acanthomatous ameloblastoma $(A, B)$ and unicystic ameloblastoma (C,D). A) Podoplanin is expressed in the outer cells (arrows) of both types of ameloblastomas, while the acanthomatous central cells (asterisk) are negative (A,C). On the other hand, ezrin presents positivity in the whole tumor parenchyma $(B, D)$. 
expressions $(\mathrm{P}=0.021)$. There was no statistical difference between membranous podoplanin and cytoplasmic ezrin expressions $(\mathrm{P}=0.285)$, as illustrated in the Table 5 .

\section{Discussion}

The podoplanin expression by epithelial cells of the benign odontogenic tumors has been recently described, ${ }^{11-21}$ but its exact biologic function in those cells remains unclear.

In recent years, our research group has been particularly interested in understanding the significance of podoplanin expression in odontogenic tumors. ${ }^{11,18}$ Its marked concentration in the outer cells of odontogenic neoplastic epithelium has lead us and other authors to postulate few theories about its function in this location, such as a possible role in the proliferative activity ${ }^{18}$ and epithelial-mesenchymal transition (EMT). ${ }^{15}$ However, both studies did not show correlation between PDPN and those activities markers expressions. Then, an interesting study performed by Martín-Villar et $a l$. demonstrated that the PDPN directly binds ezrin, activating RhoA. This linkage induces the phosphorylation of ezrin and promotes the actin cytoskeleton rearrangement in vitro. ${ }^{2}$ This finding encouraged us to investigate if PDPN and ezrin are co-located in the periphery of odontogenic benign tumors such as ameloblastomas.

In our updated samples of ameloblastomas, the podoplanin expression was very similar to the previous studies conducted in our laboratory, ${ }^{11,18}$ i.e. practically restricted to the periphery of islands and strands of epithelial odontogenic cells. Again, the central acanthomatous e reticulum-stellate like cells faintly expressed PDPN. It either corroborates other previous studies.11,15,18,19,21,22 Podoplanin expression is also observed in other odontogenic tumors, such as odontomas, ${ }^{14}$ keratocystic odontogenic tumors, calcifying cystic odontogenic tumors, ${ }^{11,19}$ and adenomatoid odontogenic tumors. ${ }^{11,21}$ In such neoplasias, its high positivity in the peripheral odontogenic epithelial cells and negativity in central and more differentiated cells and structures are likewise seen, confirming our results in the present study.

To our knowledge, this is the first report of the ezrin expression in an odontogenic tumor. In our ameloblastomas, ezrin expression was encountered in all cellular types of ameloblastoma and this positivity was consistently observed in almost all samples. Its expression included the peripheral odontogenic cells of islands and cords of odontogenic cells and reached the central portion of the tumor. Curiously, the acanthomatous and reticulumstellate-like cells also exhibited strong stain- ing of ezrin. In order to quantify and compare PDPN and ezrin expressions in the ameloblastomas, we evaluated both immunostainings in the cytoplasm and plasmatic membrane of peripheral odontogenic cells, separately, and by a scoring system. Surprisingly, despite of strong membranous and cytoplasmic PDPN and cytoplasmic ezrin expressions in the majority of tumor, there was not a positive significant correlation between their immunoexpressions by odontogenic epithelial cells $(\mathrm{P}>0.05)$. The explanation for this could be the low number of absent/weak expressed ameloblastomas. Hence, we decided to include a comparative test.

The PDPN positivity, as expected, ${ }^{11,15,18}$ was equally distributed among the cytoplasm and plasmatic membrane of ameloblastomas $(\mathrm{P}=0.157)$. The expression of ezrin was predominantly strong in the cytoplasm of the peripheral cells of the tumors. Its membranous expression, on the other hand, was absent in the majority of ameloblastomas $(\mathrm{P}=0.000)$. We found that cytoplasmic ezrin and membranous PDPN expressions were similar $(\mathrm{P}=0.285)$. In other words, both patterns of immunostaining were strongly observed in the peripheral epithelial odontogenic cells of ameloblastomas. Interestingly, Madan et al. ${ }^{28}$ and MartínVillar et $a .^{2}{ }^{2}$ demonstrated that these above cited cellular locations of expression of ezrin and PDPN refer to their active forms.

Our and others' previously published data suggested that the membranous positivity of PDPN in ameloblastomas apparently was related to the local invasion of this odontogenic tumor. ${ }^{11,18,22}$ Additionally, although ezrin expression has not been studied in odontogenic tumors, Madan et al. showed that the cytoplasmic ezrin is associated with poorer prognosis of squamous cell carcinoma (SCC) of the upper aerodigestive tract. ${ }^{28}$ Together, these data suggest a potential role of membranous PDPN and cytoplasmic ezrin in the local

Table 4. Correlation between membranous and cytoplasmic expressions of podoplanin and ezrin by peripheral cells in ameloblastomas.

\begin{tabular}{lll} 
& Membranous podoplanin & Cytoplasmic podoplanin \\
Membranous ezrin & $\mathrm{R}=-0.18$ & $\mathrm{R}=-0.21$ \\
& $\mathrm{P}=0.233$ & $\mathrm{P}=0.163$ \\
Cytoplasmic ezrin & $\mathrm{R}=-0.06$ & $\mathrm{R}=-0.14$ \\
& $\mathrm{P}=0.708$ & $\mathrm{P}=0.337$ \\
\hline
\end{tabular}

R, Spearman's rank correlation coefficient; $P$, statistical significance at $<0.05$

Table 5. Association between membranous and cytoplasmic expressions of podoplanin and ezrin in the peripheral cells in ameloblastomas.

\begin{tabular}{lcc} 
& Membranous podoplanin & Cytoplasmic podoplanin \\
Membranous ezrin & $\mathrm{P}=0.000$ & $\mathrm{P}=0.000$ \\
Cytoplasmic ezrin & $\mathrm{P}=0.285$ & $\mathrm{P}=0.021$ \\
\hline
\end{tabular}

Wilcoxon test; P, statistical significance at $<0.05$.

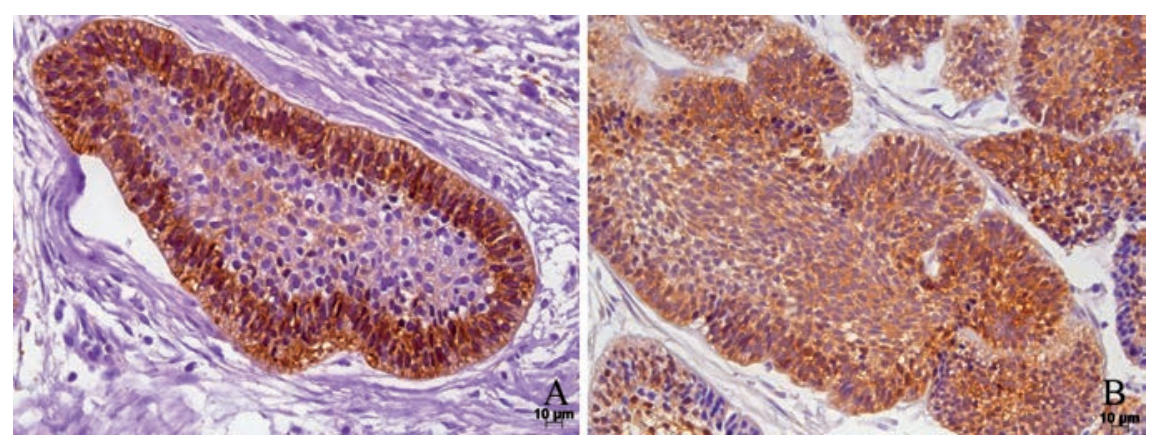

Figure 3. Immunohistochemical reactivities for podoplanin (A) and ezrin (B) in follicular ameloblastoma. This figure illustrates the strong membranous and cytoplasmic expressions of both proteins in the outer cells of the tumoral epithelial islands of ameloblastoma. 
expansion of these benign odontogenic tumors and malignant squamous cell carcinomas in the head and neck region. On the other hand, the membranous PDPN $x$ membranous ezrin, and cytoplasmic ezrin $\mathrm{x}$ cytoplasmic PDPN in the outer odontogenic cells of ameloblastomas (Table 5) showed statistically significant difference $(\mathrm{P}<0.005)$, i.e. the active forms of both antibodies were significantly more pronounced than their inactive forms. PDPN as well as ezrin are constitutively expressed in a broad variety of human tissues in physiological conditions, ${ }^{35,36}$ in order to maintain the cellular shape and polarity. When the cells present a low remodeling cytoskeletal demand, PDPN and ezrin remain, respectively, expressed in the cytoplasm and membrane of the cells or even absent. Meanwhile, when cellular movement is required, their locations shift to the plasmatic membrane (PDPN) and cytoplasm (ezrin), ${ }^{28,36}$ respectively. Thus, the strong expression of ezrin in the central as well as in the peripheral cells of ameloblastomas may be important to maintain their cellular shape. We opted to do not include the central region of the ameloblastomas in our detailed evaluation because the stellate-reticulum-like cells are often rip of the cuts due to their delicate structure and either because it is difficult to distinguish the cytoplasm and plasmatic membrane in such cells. However, the acanthomatous component of the ameloblastomas presented a markedly positivity (Figure 2B). As ezrin presents numerous binders, ${ }^{26,35}$ it is possible that this protein participates in the squamous metaplasia of the central cells associated with other partners. All the above data show that podoplanin and ezrin together are expressed in the invasive area of the odontogenic epithelium of ameloblastomas, suggesting that both proteins may have a role in the tumoral growth. In the last years, the PDPN has emerged as a potential marker of tumoral invasion, metastasis and poor prognosis of some types of cancer. ${ }^{4,6-11} \mathrm{~A}$ critical event during the tumor development is the epithelial-mesenchymal transition (EMT) which occurs when an epithelial cell loses its epithelial markers, such as E-cadherin, and begins to express mesenchymal markers, such as $\mathrm{N}$-cadherin and vimentin, decreasing their cohesiveness and acquiring migratory characteristics. ${ }^{2,3}$ It was demonstrated that podoplanin is able to mediate a particular mechanism of malignant invasion, independent of the cadherin switch and EMT. ${ }^{24}$ According to Wicki and Christofori, ${ }^{3}$ the podoplanin can induce the cellular actin cytoskeleton rearrangement binding with proteins ERM (ezrin, radixin e moesin).

The coincident expressions of podoplanin and ezrin in the peripheral odontogenic cells of ameloblastomas, together with the available clinical and in vitro data, ${ }^{2,11,18,22}$ suggest a synergic role of both proteins in the promotion of actin cytoskeletal activity, contributing to the local spread of ameloblastomas. However, further investigations employing molecular biology methods are necessary to confirm this hypothesis in odontogenic tumors.

Our results prove that podoplanin and ezrin are strongly expressed by the periphery of the tumor odontogenic epithelium and suggest a synergic role of both proteins in the process of invasion of ameloblastomas.

\section{References}

1. Astarita JL, Acton SE, Turley SJ. Podoplanin: emerging functions in development, the immune system, and cancer. Front Immunol 2012;3:283.

2. Martin-Villar E, Megias D, Castel S, Yurrita MM, Vilaro S, Quintanilla M. Podoplanin binds ERM proteins to activate RhoA and promote epithelial-mesenchymal transition. J Cell Sci 2006;119(Pt 21):4541-53.

3. Wicki A, Christofori G. The potential role of podoplanin in tumour invasion. Br $\mathrm{J}$ Cancer 2007;96:1-5.

4. Cueni LN, Chen L, Zhang H, Marino D, Huggenberger R, Alitalo A, et al. Podoplanin-Fc reduces lymphatic vessel formation in vitro and in vivo and causes disseminated intravascular coagulation when transgenically expressed in the skin. Blood 2010;116:4376-84.

5. dos Santos Almeida A, Oliveira DT, Pereira MC, Faustino SE, Nonogaki S, Carvalho AL, et al. Podoplanin and VEGF-C immunoexpression in oral squamous cell carcinomas: prognostic significance. Anticancer Res 2013;33:3969-76.

6. Huber GF, Fritzsche FR, Zullig L, Storz M, Graf N, Haerle SK, et al. Podoplanin expression correlates with sentinel lymph node metastasis in early squamous cell carcinomas of the oral cavity and oropharynx. Int J Cancer 2011;129:1404-9.

7. Kreppel M, Scheer M, Drebber U, Ritter L, Zoller JE. Impact of podoplanin expression in oral squamous cell carcinoma: clinical and histopathologic correlations. Virchows Arch 2010;456:473-82.

8. Vormittag L, Thurnher D, Geleff S, Pammer J, Heiduschka G, Brunner M, et al. Co-expression of Bmi-1 and podoplanin predicts overall survival in patients with squamous cell carcinoma of the head and neck treated with radio(chemo)therapy. Int J Radiat Oncol Biol Phys 2009;73:913-8.

9. Yuan P, Temam S, El-Naggar A, Zhou X, Liu $\mathrm{DD}$, Lee $\mathrm{JJ}$, et al. Overexpression of podoplanin in oral cancer and its association with poor clinical outcome. Cancer 2006;107:563-9.

10. Bartuli FN, Luciani F, Caddeo F, Compagni S, Piva P, Ottria L, et al. Podoplanin in the development and progression of oral cavity cancer: a preliminary study. Oral Implantol (Rome) 2012;5:33-41.

11. Caetano Ados S, Tjioe KC, Faustino SE, Hanemann JA, Belone Ade F, Soares CT, et al. Immunolocalization of podoplanin in benign odontogenic tumours with and without ectomesenchyme. Arch Oral Biol 2013;58:408-15.

12. Friedrich RE, Scheuer HA, Zustin J. Expression of podoplanin in nevoid basal cell carcinoma syndrome-associated keratocystic odontogenic tumours. Anticancer Res 2012;32:2125-7.

13. Friedrich RE, Zustin J. Calcifying epithelial odontogenic tumour of the maxilla: a case report with respect to immunohistochemical findings. In Vivo 2011;25:259-64.

14. Gonzalez-Alva P, Inoue H, Miyazaki Y, Tsuchiya H, Noguchi Y, Kikuchi K, et al. Podoplanin expression in odontomas: clinicopathological study and immunohistochemical analysis of 86 cases. J Oral Sci 2011;53:67-75.

15. Gonzalez-Alva P, Tanaka A, Oku Y, Miyazaki Y, Okamoto E, Fujinami M, et al. Enhanced expression of podoplanin in ameloblastomas. J Oral Pathol Med 2010;39:103-9.

16. Kikuchi K, Ito $\mathrm{S}$, Inoue $\mathrm{H}$, Gonzalez-Alva $\mathrm{P}$, Miyazaki Y, Sakashita $\mathrm{H}$, et al. Immunohistochemical expression of podoplanin in so-called hard alpha-keratin-expressing tumors, including calcifying cystic odontogenic tumor, craniopharyngioma, and pilomatrixoma. J Oral Sci 2012;54:165-75.

17. Okamoto E, Kikuchi K, Miyazaki Y, Gonzalez-Alva P, Oku Y, Tanaka A, et al. Significance of podoplanin expression in keratocystic odontogenic tumor. J Oral Pathol Med 2010;39:110-4.

18. Tjioe KC, Oliveira DT, Soares CT, Lauris JR, Damante JH. Is podoplanin expression associated with the proliferative activity of ameloblastomas? Oral Dis 2012;18:673-9.

19. Tsuneki M, Maruyama S, Yamazaki M, Cheng J, Saku T. Podoplanin expression profiles characteristic of odontogenic tumor-specific tissue architectures. Pathol Res Pract 2012;208:140-6.

20. Zhang X, Wang J, Ding X, Xing S, Zhang W, Wang $L$, et al. Altered expression of podoplanin in keratocystic odontogenic tumours following decompression. Oncol Lett 2014;7:627-30.

21. Zustin J, Scheuer HA, Friedrich RE. Podoplanin expression in human tooth 
germ tissues and cystic odontogenic lesions: an immunohistochemical study. J Oral Pathol Med 2010;39:115-20.

22. Siar CH, Ishak I, Ng KH. Podoplanin, Ecadherin, beta-catenin, and CD44v6 in recurrent ameloblastoma: their distribution patterns and relevance. J Oral Pathol Med 2015;44:51-8.

23. Fernandez-Munoz B, Yurrita MM, MartinVillar E, Carrasco-Ramirez P, Megias D, Renart J, et al. The transmembrane domain of podoplanin is required for its association with lipid rafts and the induction of epithelial-mesenchymal transition. Int J Biochem Cell Biol 2011;43:886-96.

24. Wicki A, Lehembre F, Wick N, Hantusch B, Kerjaschki D, Christofori G. Tumor invasion in the absence of epithelial-mesenchymal transition: podoplanin-mediated remodeling of the actin cytoskeleton. Cancer Cell 2006;9:261-72.

25. Bretscher A, Edwards K, Fehon RG. ERM proteins and merlin: integrators at the cell cortex. Nat Rev Mol Cell Biol 2002;3:586-99.

26. Fehon RG, McClatchey AI, Bretscher A. Organizing the cell cortex: the role of ERM proteins. Nat Rev Mol Cell Biol 2010;11:276-87.

27. Revenu C, Athman R, Robine S, Louvard D. The co-workers of actin filaments: from cell structures to signals. Nat Rev Mol Cell Biol 2004;5:635-46.

28. Madan R, Brandwein-Gensler M, Schlecht NF, Elias K, Gorbovitsky E, Belbin TJ, et al. Differential tissue and subcellular expressionof ERM proteins in normal and malignant tissues: cytoplasmic ezrin expression has prognostic signficance for head and neck squamous cell carcinoma. Head Neck 2006;28:1018-27.

29. Mhawech-Fauceglia P, Dulguerov P, Beck A, Bonet M, Allal AS. Value of ezrin, maspin and nm23-H1 protein expressions in predicting outcome of patients with head and neck squamous-cell carcinoma treated with radical radiotherapy. J Clin Pathol 2007;60:185-9.

30. Saito S, Yamamoto H, Mukaisho K, Sato S, Higo T, Hattori T, et al. Mechanisms underlying cancer progression caused by ezrin overexpression in tongue squamous cell carcinoma. PLoS One 2013;8:e54881.

31. Schlecht NF, Brandwein-Gensler M, Smith RV, Kawachi N, Broughel D, Lin J, et al. Cytoplasmic ezrin and moesin correlate with poor survival in head and neck squamous cell carcinoma. Head Neck Pathol 2012;6:232-43.

32. Barnes L, Eveson JW, Reichart P, Sidransky D. Ameloblastomas, p. 296-7. World Health
Organization Classification of Tumours Pathology and genetics - Head and neck tumours. IAR Press, Lyon, 2005.

33. Piffko J, Bankfalvi A, Ofner D, Rasch D, Joos U, Schmid KW. Standardized demonstration of silver-stained nucleolar organizer regions-associated proteins in archival oral squamous cell carcinomas and adjacent non-neoplastic mucosa. Mod Pathol 1997;10:98-104.

34. Soini Y, Puhakka A, Kahlos K, Saily M, Paakko P, Koistinen P, et al. Endothelial nitric oxide synthase is strongly expressed in malignant mesothelioma but does not associate with vascular density or the expression of VEGF, FLK1 or FLT1. Histopathology 2001;39:179-86.

35. Arpin M, Chirivino D, Naba A, Zwaenepoel I. Emerging role for ERM proteins in cell adhesion and migration. Cell Adh Migr 2011;5:199-206.

36. Martin-Villar E, Scholl FG, Gamallo C, Yurrita MM, Munoz-Guerra M, Cruces J, et al. Characterization of human PA2.26 antigen (T1alpha-2, podoplanin), a small membrane mucin induced in oral squamous cell carcinomas. Int J Cancer 2005; 113:899-910. 